\title{
Subclinical infection without encephalitis in mice following intranasal exposure to Nipah virus-Malaysia and Nipah virus-Bangladesh
}

\author{
Johanna Dups ${ }^{1}$, Deborah Middleton ${ }^{1}$, Fenella Long ${ }^{1}$, Rachel Arkinstall ${ }^{1}$, Glenn A Marsh ${ }^{1 *}$ and Lin-Fa Wang ${ }^{1,2}$
}

\begin{abstract}
Background: Nipah virus and Hendra virus are closely related and following natural or experimental exposure induce similar clinical disease. In humans, encephalitis is the most serious outcome of infection and, hitherto, research into the pathogenesis of henipavirus encephalitis has been limited by the lack of a suitable model. Recently we reported a wild-type mouse model of Hendra virus ( $\mathrm{HeV}$ ) encephalitis that should facilitate detailed investigations of its neuropathogenesis, including mechanisms of disease recrudescence. In this study we investigated the possibility of developing a similar model of Nipah virus encephalitis.

Findings: Aged and young adult wild type mice did not develop clinical disease including encephalitis following intranasal exposure to either the Malaysia (NiV-MY) or Bangladesh (NiV-BD) strains of Nipah virus. However viral RNA was detected in lung tissue of mice at euthanasia (21 days following exposure) accompanied by a non-neutralizing antibody response. In a subsequent time course trial this viral RNA was shown to be reflective of an earlier self-limiting and subclinical lower respiratory tract infection through successful virus re-isolation and antigen detection in lung. There was no evidence for viremia or infection of other organs, including brain.

Conclusions: Mice develop a subclinical self-limiting lower respiratory tract infection but not encephalitis following intranasal exposure to NiV-BD or NiV-MY. These results contrast with those reported for HeV under similar exposure conditions in mice, demonstrating a significant biological difference in host clinical response to exposure with these viruses. This finding provides a new platform from which to explore the viral and/or host factors that determine the neuroinvasive ability of henipaviruses.
\end{abstract}

Keywords: Henipavirus, Hendra virus, Nipah virus, Encephalitis, Mouse, Mice, Model

\section{Introduction}

Nipah virus (NiV; genus Henipavirus, family Paramyxoviridae) is closely related to Hendra virus (HeV; genus Henipavirus, family Paramyxoviridae) [1] and both cause severe and often fatal encephalitic disease in humans $[2,3]$. We recently reported a wild-type mouse model of $\mathrm{HeV}$ encephalitis [4]; a particularly useful model for this aspect of the disease as, in contrast to many other animal models, mice neither develop systemic infection nor succumb to acute disease prior to establishment of encephalitis. In addition there is

\footnotetext{
* Correspondence: glenn.marsh@csiro.au

'CSIRO Animal, Food and Health Science, Australian Animal Health Laboratory, Geelong, VIC 3219, Australia

Full list of author information is available at the end of the article
}

excellent access to reagents for more detailed investigation of the brain disease in this species. We were interested to determine whether a similar model of $\mathrm{NiV}$ encephalitis in the mouse could be developed given that both viruses use the same host receptor [5,6] and are observed to cause similar clinical disease in several species (reviewed in [7] and [8]).

Nipah virus infection of mice has been investigated previously $[9,10]$. These studies showed that young adult wild-type mice exposed to Nipah virus using the intranasal or intraperitoneal route did not develop clinical disease. However, effects of mouse strain and age were not described and neither was subclinical infection, which is now known to occur regularly in young adult mice after 
intranasal $\mathrm{HeV}$ exposure [4]. Susceptibility of mice to the Bangladesh strain of NiV has not been reported.

Here we investigate NiV strains from Malaysia (NiV-MY) and Bangladesh (NiV-BD) in infection of young adult and aged mice of two strains (BALB/c and C57BL/6). Both these mouse strains are known to be susceptible to the closely related Hendra virus, with aged mice reliably developing clinical disease [4].

Table 1 Assessment of specific (binding) antibody to NiV soluble-G and neutralising antibody at day 21 post-exposure

\begin{tabular}{|c|c|c|c|c|c|}
\hline Virus strain & Mouse strain & Age & Mouse \# & Binding antibody to NiV sG^ & Serum neutralisation \\
\hline \multirow[t]{18}{*}{ Nipah Malaysia } & $\mathrm{BALB} / \mathrm{C}$ & $Y A^{*}$ & 1 & + & - \\
\hline & & & 2 & + & - \\
\hline & & & 3 & + & - \\
\hline & & & 4 & + & - \\
\hline & & & 5 & + & - \\
\hline & & Aged & 6 & + & - \\
\hline & & & 7 & - & - \\
\hline & & & 8 & + & - \\
\hline & & & 9 & + & - \\
\hline & & & 10 & + & - \\
\hline & C57BL/6 & YA & 11 & + & - \\
\hline & & & 12 & + & - \\
\hline & & & 13 & + & - \\
\hline & & & 14 & + & - \\
\hline & & Aged & 15 & + & - \\
\hline & & & 16 & + & - \\
\hline & & & 17 & - & - \\
\hline & & & 18 & + & - \\
\hline \multirow[t]{19}{*}{ Nipah Bangladesh } & $\mathrm{BALB} / \mathrm{C}$ & YA & 19 & + & - \\
\hline & & & 20 & + & - \\
\hline & & & 21 & + & - \\
\hline & & & 22 & + & - \\
\hline & & & 23 & - & - \\
\hline & & Aged & 24 & - & - \\
\hline & & & 25 & + & - \\
\hline & & & 26 & + & - \\
\hline & & & 27 & + & - \\
\hline & & & 28 & + & - \\
\hline & C57BL/6 & YA & 29 & - & - \\
\hline & & & 30 & - & - \\
\hline & & & 31 & + & - \\
\hline & & & 32 & - & - \\
\hline & & & 33 & + & - \\
\hline & & Aged & 34 & - & - \\
\hline & & & 35 & - & - \\
\hline & & & 36 & - & - \\
\hline & & & 37 & - & - \\
\hline
\end{tabular}




\section{Experimental design, methods and findings}

To study NiV infection in mice, we exposed young adult (8 weeks) and aged (12 months) BALB/c and C57BL/6 mice to 50,000 TCID $_{50}$ of low passage human isolates of NiV-MY (Nipah virus/Malaysia/human/99) or NiV-BD (Nipah Bangladesh/human/2004/Rajbari,R1) by the intranasal route (BALB/c $n=5$ all groups, C57BL/6 $n=4$ all groups except young adult NiV-BD $n=5)$. Mice were monitored daily and euthanased at onset of clinical signs or at 21 days post challenge. All animal work was approved by the CSIRO Australian Animal Health Laboratory Animal Ethics Committee.

All mice remained free of clinical disease for the period of observation and were euthanased at day 21 . However, consistent with an adaptive immune response to viral replication, many mice were positive for specific binding antibody to the soluble form of NiV G glycoprotein (NiV sG) as detected by Luminex microsphere assay [11] (Table 1). Neutralising antibodies were not detected by serum neutralisation assay.

To explore the possibility of subclinical NiV infection, we analysed brain, lung, heart, spleen, liver, kidney, mesenteric lymph nodes, ovaries and blood collected at euthanasia (day 21) for presence of viral RNA (vRNA) by real time PCR [12], lesions by histopathology and viral antigen by immunohistochemistry. We attempted virus isolation on all tissues positive for vRNA. All analyses were performed as previously described [4].

We did not detect NiV vRNA (Figure 1), viral antigen, or lesions of encephalitis in any mouse brain. However, lung tissue of several mice tested positive for NiV-MY and NiV-BD vRNA (Figure 1), without pulmonary lesions or demonstrable viral antigen. BALB/c aged mice exposed to NiV-BD had higher levels of viral genome in lungs (quantified by comparison to a standard curve to calculate copy number) than any other groups (Figure 1).
Remaining tissues were negative for all tests described above except for the detection of vRNA in two cases only (Figure 1), suggesting that systemic infection had not occurred. Of note, these two mice were not positive for vRNA in lung tissue.

To further assess whether the NiV vRNA detected in lungs at 21 days reflected an earlier self-limiting respiratory tract infection, we performed a time course study in aged BALB/c mice exposed intranasally to 50,000 TCID $_{50}$ NiV-MY or NiV-BD. Two mice for each virus strain were euthanased at 48 hour intervals up to day 12 post exposure and at day 15 post exposure.

As expected, animals did not develop clinical disease and were euthanased at the pre-determined time points. Samples were collected and analysed as described above.

We detected viral RNA in lung tissue at levels ranging from $1.2-4.4 \log _{10}$ copies $/ 10^{12}$ copies $18 \mathrm{~s}$ rRNA at all sampled time points for both virus strains (Figure 2). NiV-MY and -BD were isolated inconsistently from RNA-positive lung tissues using the method described previously (4). To increase test sensitivity $100 \mathrm{ul}$ of supernatant from tissue homogenate was used to inoculate a sub-confluent well of Veros (6 well plate) and reisolation was successful up to day 10 post challenge (Figure 2). Viral titres were consistently low $\left(<40 \mathrm{TCID}_{50} / \mathrm{ml}\right)$. Viral antigen was detected by immunohistochemistry in the alveolar walls of mice infected with NiV-BD and NiVMY (Figure 3), with mice given NiV-BD significantly more likely to show a positive reaction $(\mathrm{p}=0.04$, Fisher's exact test): it was not possible to distinguish on morphologic grounds whether alveolar lining cells, alveolar interstitium, or endothelial cells were involved. Pneumonia was not identified in any mouse. Viremia was not detected at any time point and all remaining tissues including brain were negative for vRNA, viral antigen and viral genome, except for ovary, thymus and heart of one NiV-MY challenged

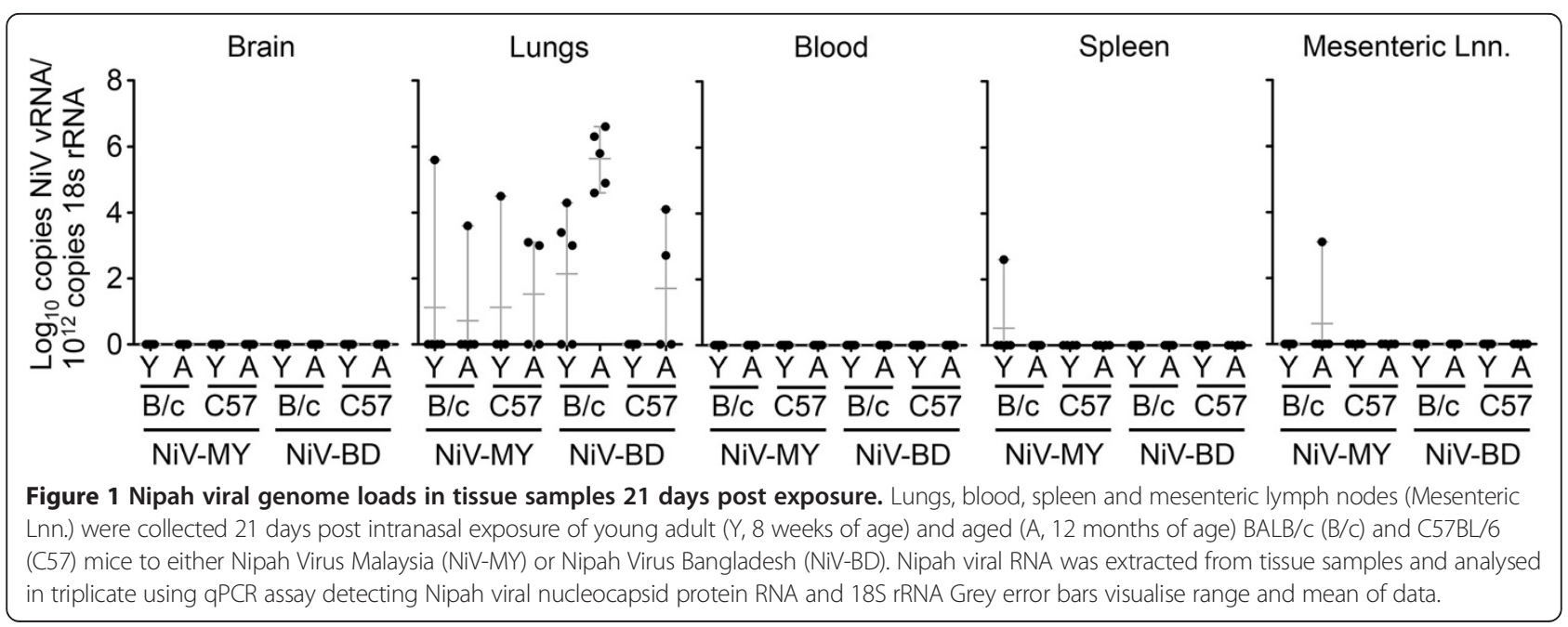




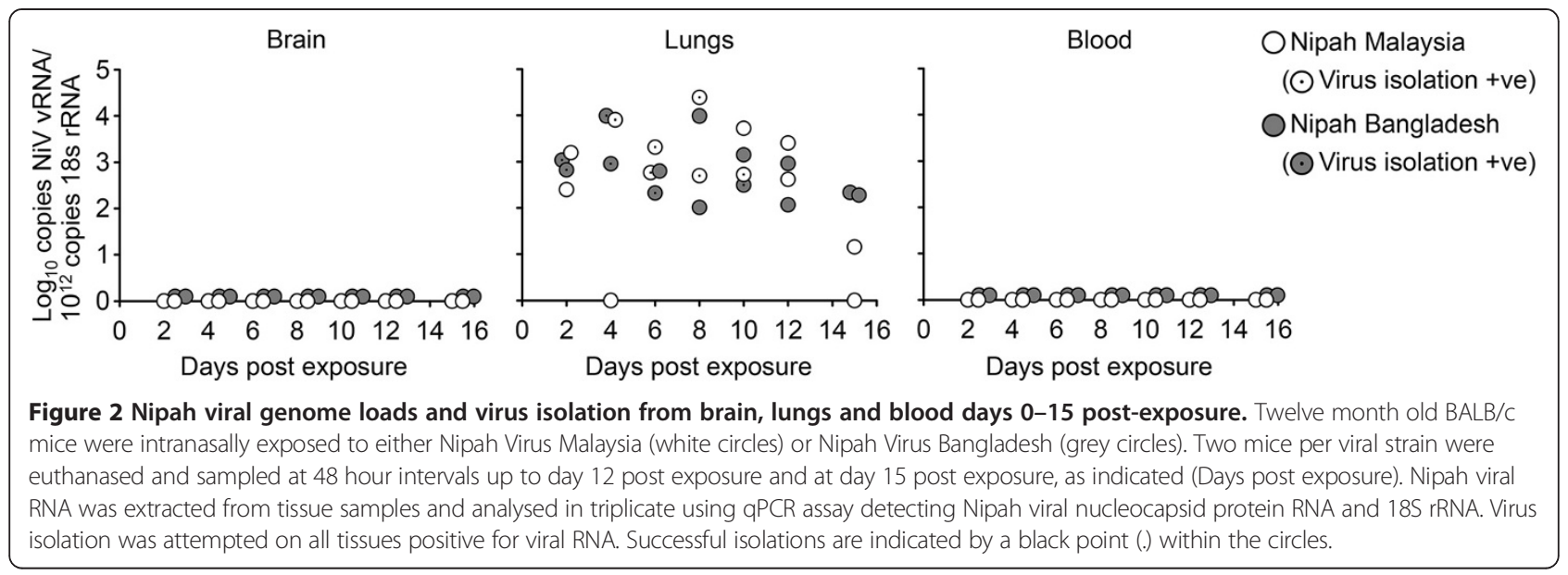

mouse and cervical lymph nodes of two NiV-BD challenged mice which were positive for viral genome but not for antigen nor for lesions.

To test for infection in the upper respiratory tract we examined the nasal cavity of each mouse for evidence of local rhinitis and presence of NiV antigen by histopathology and immunohistochemistry. Viral antigen and lesions consistent with rhinitis were not detected except in one NiV-BD study animal euthanased 10 days postexposure.

Taken together, these results demonstrate that mice exposed to $50,000 \mathrm{TCID}_{50}$ NiV-BD or NiV-MY develop a subclinical, self-limiting lower respiratory tract infection without encephalitis or systemic infection.

\section{Conclusion and discussion}

The observation of sub-clinical self-limiting lower respiratory tract infection without encephalitis in wildtype mice exposed to NiV-MY and NiV-BD contrasted with certain reported findings using $\mathrm{HeV}$ in mice under similar exposure conditions [4]. In that report, aged $\mathrm{BALB} / \mathrm{c}$ and C57BL/6 mice reliably developed neurological disease following intranasal exposure to $\mathrm{HeV}$, with sub-clinical encephalitis also documented in young adult mice. In addition, NiV-MY and -BD were reisolated from RNA-positive lung tissues less consistently compared to $\mathrm{HeV}$ using the method described previously and at lower titres, with levels of $\mathrm{HeV}$ reported up to $200 \mathrm{TCID}_{50} / \mathrm{ml}$. Considered together, the data suggest that - unlike what has been observed in other permissive animal hosts - NiV infection of mice is less productive than $\mathrm{HeV}$ and there is a categorical difference in the pathogenicity of $\mathrm{HeV}$ and $\mathrm{NiV}$ for mice. In particular, $\mathrm{NiV}$ appears unable to establish infection of the central nervous system via the olfactory receptor neuron pathway as observed for $\mathrm{HeV}$. Future in vitro studies characterising infection dynamics of $\mathrm{NiV}$ and $\mathrm{HeV}$ in specific cell types of the olfactory epithelium will help to identify the mechanisms involved in inhibition of NiV neuroinvasion.

Increased levels of viral genome were detected in the lungs of aged $\mathrm{BALB} / \mathrm{c}$ mice exposed to NiV-BD at 21 days compared to other groups (Figure 1). This may be explained by increased virus production during the replication phase of infection or a decreased ability to clear residual viral material. We found comparable levels of viral genome during the period in which replication was observed (up to day 10 post exposure), in aged BALB/c mice following exposure to either NiV-BD or

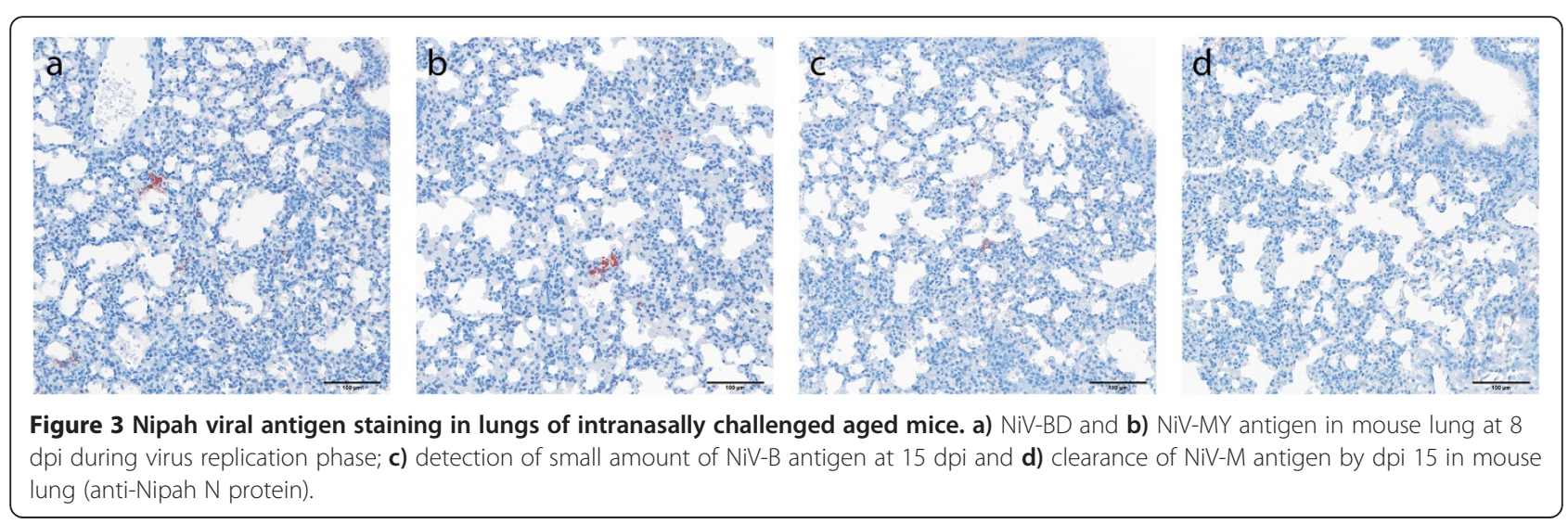


NiV-MY (Figure 2) ruling out the first explanation. The possibility of less efficient clearance of NiV-BD by aged $\mathrm{BALB} / \mathrm{c}$ mice requires further investigation.

Nipah virus infection detected in lung tissue appeared controlled at this location and did not spread systemically, as viremia was not detected and there was no evidence for productive infection of other systemic organs. Occasional tissues outside of lung were positive for viral genome but not for virus isolation, presence of antigen or presence of lesions and accordingly interpreted as not infected. Control and resolution of the lower respiratory tract infection was not the result of the development of neutralizing antibodies as these were not detected in any mouse at 21 days post-exposure. Similar to $\mathrm{HeV}$, nonneutralising binding antibodies against $\mathrm{NiV}$ sG were detected by Luminex microsphere assay, however, their role in control or clearance of infection is unclear and requires further investigation.

Comparing to that observed with $\mathrm{HeV}$ in mice [4], the combined infection models elicit a significant biological difference between $\mathrm{HeV}$ and $\mathrm{NiV}$ at the host level. Under matching conditions and unlike $\mathrm{HeV}$, exposure to $\mathrm{NiV}$ lead to infection but not to encephalitis in mice and, as the same mouse strains of the same ages were used in the two studies, it is likely that viral factors (of which dose is one possibility) rather than host factors are responsible for this. Neuroinvasion progressing to encephalitis is the most serious outcome of human infections with henipaviruses: the susceptibility of mice to $\mathrm{HeV}$ encephalitis but not $\mathrm{NiV}$ encephalitis at equivalent dose presents a key that can be used to define the determinants of henipavirus neuropathogenesis in mice. This information will likely have implications for the development of therapies that can be applied to manage the important neurological complications of human infections.

\section{Competing interests}

The authors declare that they have no competing interests.

\section{Author details}

'CSIRO Animal, Food and Health Science, Australian Animal Health Laboratory, Geelong, VIC 3219, Australia. ${ }^{2}$ Duke-NUS Graduate Medical School, 21 Lower Kent Ridge Road, Singapore 119077, Singapore.

Received: 7 April 2014 Accepted: 23 May 2014

Published: 2 June 2014

\section{References}

1. Wang L, Harcourt BH, Yu M, Tamin A, Rota PA, Bellini WJ, Eaton BT: Molecular biology of Hendra and Nipah viruses. Microbes Infect 2001, 3:279-287.

2. Playford EG, McCall B, Smith G, Slinko V, Allen G, Smith I, Moore F, Taylor C, Kung YH, Field H: Human Hendra virus encephalitis associated with equine outbreak, Australia, 2008. Emerg Infect Dis 2010, 16:219-223.

3. Tan CT, Wong KT: Nipah encephalitis outbreak in Malaysia. Ann Acad Med Singapore 2003, 32:112-117.

4. Dups J, Middleton D, Yamada M, Monaghan P, Long F, Robinson R, Marsh GA, Wang LF: A new model for hendra virus encephalitis in the mouse. PLoS One 2012, 7:e40308

5. Bonaparte MI, Dimitrov AS, Bossart KN, Crameri G, Mungall BA, Bishop KA, Choudhry V, Dimitrov DS, Wang LF, Eaton BT, Broder CC: Ephrin-B2 ligand is a functional receptor for Hendra virus and Nipah virus. Proc Natl Acad Sci U S A 2005, 102:10652-10657.

6. Negrete $\mathrm{OA}$, Chu D, Aguilar HC, Lee B: Single amino acid changes in the Nipah and Hendra virus attachment glycoproteins distinguish ephrinB2 from ephrinB3 usage. J Virol 2007, 81:10804-10814.

7. Geisbert TW, Feldmann H, Broder CC: Animal challenge models of henipavirus infection and pathogenesis. Curr Top Microbiol Immunol 2012, 359:153-177.

8. Middleton DJ, Weingartl HM: Henipaviruses in their natural animal hosts. Curr Top Microbiol Immunol 2012, 359:105-121.

9. Wong KT, Grosjean I, Brisson C, Blanquier B, Fevre-Montange M, Bernard A, Loth P, Georges-Courbot MC, Chevallier M, Akaoka H, Marianneau P, Lam SK, Wild TF, Deubel V: A golden hamster model for human acute Nipah virus infection. Am J Pathol 2003, 163:2127-2137.

10. Dhondt KP, Mathieu C, Chalons M, Reynaud JM, Vallve A, Raoul H, Horvat B: Type I interferon signaling protects mice from lethal Henipavirus infection. J Infect Dis 2012, 207(1):142-151.

11. Bossart KN, McEachern JA, Hickey AC, Choudhry V, Dimitrov DS, Eaton BT, Wang LF: Neutralization assays for differential henipavirus serology using Bio-Plex protein array systems. J Virol Methods 2007, 142:29-40.

12. Feldman KS, Foord A, Heine HG, Smith IL, Boyd V, Marsh GA, Wood JL, Cunningham AA, Wang LF: Design and evaluation of consensus PCR assays for henipaviruses. J Virol Methods 2009, 161:52-57.

doi:10.1186/1743-422X-11-102

Cite this article as: Dups et al:: Subclinical infection without encephalitis in mice following intranasal exposure to Nipah virus-Malaysia and Nipah virus-Bangladesh. Virology Journal 2014 11:102.

\begin{abstract}
Authors' contributions
JD conceived the study, carried out the animal infection trials and sampling, processed and analysed samples and drafted the manuscript. DM conceived the study, participated in study design, analysed samples and helped to draft the manuscript. FL carried out histopathological and immunohistochemical analyses. RA carried out the animal infection trials. GM conceived of the study, participated in design of the study and helped to draft the manuscript. LW conceived of the study, participated in design of the study and helped to draft the manuscript. All authors read and approved the final manuscript.
\end{abstract}

\section{Acknowledgements}

We thank Christopher Broder (Uniformed Services University, Maryland, USA) for the supply of soluble NiV G glycoprotein. We thank Jean Payne and Jenni Harper for technical assistance, John White for assisting with RNA extractions and Leah Frazer for animal husbandry and technical help. This work was supported by The Commonwealth Scientific and Industrial Research Organisation (Science Leader funding to LFW) and the Australian National Health and Medical Research Council (grant 1022516).

\section{Submit your next manuscript to BioMed Central and take full advantage of:}

- Convenient online submission

- Thorough peer review

- No space constraints or color figure charges

- Immediate publication on acceptance

- Inclusion in PubMed, CAS, Scopus and Google Scholar

- Research which is freely available for redistribution 